\title{
Naissance et unité du patronat associatif du secteur de l'animation
}

Emergence and Alignment of a Non Profit Employers' Union in the French Sector of Community Centers

\section{Simon Cottin-Marx}

\section{CpenEdition}

Journals

\section{Édition électronique}

URL : https://journals.openedition.org/travailemploi/10663

DOI : 10.4000/travailemploi. 10663

ISSN : 1775-416X

Éditeur

DARES - Ministère du Travail

Édition imprimée

Date de publication : 15 décembre 2020

Pagination : 83-104

ISSN : 0224-4365

\section{Référence électronique}

Simon Cottin-Marx, « Naissance et unité du patronat associatif du secteur de l'animation », Travail et Emploi [En ligne], 163 | 2020, mis en ligne le 01 février 2022, consulté le 26 février 2022. URL : http:// journals.openedition.org/travailemploi/10663; DOI : https://doi.org/10.4000/travailemploi.10663 


\title{
Naissance et unité du patronat associatif du secteur de l'animation*
}

\author{
Simon Cottin-Marx ${ }^{* *}$
}

\begin{abstract}
Dans cette contribution à la sociologie du monde associatif et du patronat, nous nous intéressons à la fondation du syndicat patronal de la branche « associative» de l'animation socioculturelle. Pour la comprendre, le texte revient sur la genèse du secteur, souligne l'action déterminante de l'État dans sa salarisation et la création d'une branche professionnelle. Face à cette volonté publique, les principales entreprises associatives de l'animation vont s'organiser et un espace patronal va émerger. Après s'être divisé au gré de différentes crises, il s'unifie avec la création d'un syndicat employeur unique: le Conseil national des employeurs associatifs (CNEA), qui prend le nom d'Hexopée fin 2020. L'article montre le rôle de l'État dans la création du patronat associatif, tout en donnant à voir l'action des entreprises associatives, dont l'activité s'inscrit dans des histoires et des intérêts qui leur sont propres.
\end{abstract}

T 'émergence de syndicats patronaux dans le monde associatif est récente. À l'exception de ceux du secteur sanitaire et social (CHAUVIÈRE, 2005), la majeure partie est créée entre les années 1970 et 2000. Et ce n'est seulement qu'en 2017 que l'Union des employeurs de l'économie sociale et solidaire (UDES), qui rassemble la majorité d'entre eux, est reconnue comme une organisation professionnelle représentative au niveau «multi-professionnel » (OFFERLÉ, 2018). Comment expliquer cette émergence tardive?

Les auteurs de la sociologie du travail associatif mettent en avant le fait que la salarisation du monde associatif est relativement récente. Au début des années 1980, les « entreprises associatives » n'employaient que 615000 salariés (MARCHAL, 1989). Ils sont le triple aujourd'hui (TCHERnOnOG, Prouteau, 2019). Nous faisons donc l'hypothèse d'une corrélation entre développement de l'emploi et création

\footnotetext{
* L'auteur tient à remercier l'Institut national de la jeunesse et de l'éducation populaire (Injep) et l'Institut français du monde associatif qui ont permis la réalisation de ce post-doctorat sur le monde du travail associatif.

** Laboratoire techniques, territoires et sociétés (Latts, UMR 8134), Université Gustave Eiffel; Simon.cottin-marx@ enpc.fr.
} 
d'organisations patronales. Plusieurs travaux universitaires soulignent cependant les obstacles, spécifiques aux «entreprises associatives » (MARCHAL, 1992; HéLY, 2009), qui non seulement freinent l'émergence d'un patronat associatif, mais aussi et plus particulièrement, freinent les employeurs associatifs pour se constituer « en patronat » (OFFERLÉ, 2015). Ils mettent en avant les difficultés de ces derniers à se penser comme « employeurs ». En effet, les dirigeants des structures loi $1901^{1}$ ne sont pas «patrons » au sens strict du terme: ils ne sont pas propriétaires des moyens de production. Contrairement aux entreprises privées « classiques », les entreprises associatives ne sont pas dirigées par des détenteurs de capitaux, mais par les bénévoles qui composent leurs instances (bureau, conseil d'administration, assemblée générale, etc.). Pour ces bénévoles, employeurs car administrateurs de leurs structures, la gestion des emplois n'est pas au fondement de leur engagement ; la fonction employeur est un effet collatéral de leur engagement pour le projet. Les responsabilités qu'ils endossent dans ce cadre ne « vont pas de soi » (CARDOSO, 2019), apparaissent souvent comme inattendues, difficiles à assumer (COTTIN-MARX, 2021a) et sont rarement une priorité. S'ils se sont engagés dans leur structure, c'est d'abord pour conduire un projet, et non pour devenir employeur (CotTin-Marx, 2020; UrasadetTan, Schmidt, 2020). Comme le constatent Anne Le Roy, Emmanuelle Puissant et al. (2019, p. 69), « les employeurs associatifs sont souvent des militants qui ont choisi ou accepté d'endosser les responsabilités d'employeurs pour mieux défendre leur "cause" ». Certains dirigeants associatifs sont même parfois adhérents, ou sont d'anciens militants, de syndicats de salariés (SimONET, 2015). Autant de spécificités qui font des dirigeants associatifs des employeurs à part, pour lesquels le registre de l'engagement se superpose à celui de l'entreprise. Ainsi, la création de syndicats patronaux, pour représenter ces entreprises associatives dirigées par des bénévoles, n'a rien d'évidente.

Pour la sociologie du patronat, l'absence d'évidence n'est pas propre au monde du travail associatif. Comme l'écrit Annette Jobert (2012, p. 526), « alors que, face au pouvoir patronal, on voit bien les raisons pour lesquelles les salariés subordonnés ont cherché à se coaliser, celles qui poussent les patrons à s'organiser collectivement suscitent beaucoup d'interrogations ». Si Cornelia Woll (2006, p. 258) souligne que ce qui incite « les employeurs à l'action collective, c'est l'opposition à deux menaces : l'interventionnisme étatique [sur le marché] et le mouvement ouvrier », ces explications n'épuisent pas le sujet, ne permettent pas de comprendre pourquoi les employeurs se sont constitués en groupe d'intérêt, qui plus est hétérogène (BuNEL, SAGLIO, 1979). Pour Michel OfFerLé (2009, p. 15), il n'y a pas de « vrai précurseur » au patronat et de multiples raisons concourent à la mobilisation des chefs d'entreprise : par exemple, la « volonté de maximiser par l'action collective leur position sur le marché économique, de combattre les révolutionnaires, de faire affaire, de produire de

1. «L'association est la convention par laquelle deux ou plusieurs personnes mettent en commun, d'une façon permanente, leurs connaissances ou leur activité dans un but autre que de partager des bénéfices. Elle est régie, quant à sa validité, par les principes généraux du droit applicables aux contrats et obligations » (article 1 de la loi WaldeckRousseau du $1^{\text {er }}$ juillet 1901). 
la sociabilité, de s'imposer sur le marché politique ou d'obéir à une nécessité éthique d'agir collectivement». Cette mobilisation peut aussi être «extérieure », venir « de certains segments de l'État agissant comme producteur de catégories et d'identité » (p. 21), des législateurs qui produisent des demandes de représentation, etc.

L'étude des conditions d'émergence du syndicalisme patronal associatif est un champ de recherche encore peu exploré. La plupart des travaux se sont intéressés aux efforts de l'UDES pour intégrer le giron patronal et aux stratégies déployées par ce syndicat pour obtenir sa représentativité (MAGGI-Germain et al. 2011; CAILlaud, 2012; HÉly et al., 2014; CotTIN-MARX, HÉly, 2021), en particulier celle qui lui a permis de faire reconnaître sa légitimité par sa participation aux élections prud'homales (Michel, WillemeZ, 2009; Fraizy, 2016).

Dans cet article, nous nous intéressons à l'histoire du syndicat employeur d'un secteur emblématique du monde associatif, car héritier et porteur du puissant imaginaire de l'éducation populaire (Poujol, 1981; MignON, 2007): celui de l'animation socioculturelle. Le Conseil national des employeurs associatifs (CNEA, qui a pris le nom d'Hexopée en 2020) a été créé en 2005 par les principales associations employeuses de ce secteur, mettant ainsi un terme à plus d'une vingtaine d'années de divisions et de concurrences pour la représentation patronale.

Le CNEA est, au moment de notre enquête courant 2020, l'unique syndicat employeur représentatif dans la branche de l'animation ${ }^{2}$ et contribue à ce titre à sa structuration institutionnelle. Selon sa convention collective, la branche de l'animation regroupe les organisations qui « développent à titre principal des activités d'intérêt social dans les domaines culturels, éducatifs, de loisirs et de plein air, notamment par des actions continues ou ponctuelles d'animation, de diffusion ou d'information créatives ou récréatives ouvertes à toute catégorie de population ». Elle comprend par exemple les centres de loisirs et de vacances pour mineurs, les classes de découverte, les structures d'accueil post et périscolaire.

Outre cette branche associative où il a le monopole patronal, le CNEA est également reconnu comme syndicat représentatif dans différentes branches professionnelles où les entreprises associatives sont majoritaires. C'est le cas dans la branche $\mathrm{du}$ sport et dans celle du tourisme social et familial. Alors que dans la première, le Conseil social du mouvement sportif (CoSMoS) (FLeuriel, 2013; 2016; 2021) est majoritaire, dans la seconde, c'est le Groupement syndical des organismes de tourisme familial (GSOTF). Dans la branche des foyers de jeunes travailleurs, le CNEA est, depuis 2017, l'unique syndicat employeur représentatif. Au total, cette organisation déclare 13300 « entreprises associatives » adhérentes ${ }^{3}$, employant 145000 personnes

2. En sont exclus les animateurs employés par les collectivités locales, mais aussi ceux de la branche des acteurs du lien social et familial, etc.

3. Plusieurs fédérations, confédérations et réseaux adhèrent et incitent leurs associations à adhérer au CNEA. C'est le cas des Centres d'entraînement aux méthodes d'éducation active (CEMÉA), de la Confédération des Maisons de la jeunesse et de la culture (MJC), de la Fédération nationale des MJC, des Éclaireuses Éclaireurs de France (EEDF), de la Fédération des Francas (Francs et franches camarades), de la Fédération Léo-Lagrange, de la Fédération des 
travaillant très majoritairement $(90 \%)$ dans de très petites structures (moins de dix salariés). Elle est aussi l'un des principaux membres de l'UDES, que le président fondateur du CNEA présida de 2005 à 2015.

Pourquoi s'est structuré cet acteur patronal ? Pour répondre à cette question (encadré 1), dans la première partie de cet article, nous nous intéressons à l'histoire du secteur de l'animation socioculturelle et au processus qui a conduit à la création d'une branche dédiée. Dans la seconde partie, nous retraçons le processus d'émergence, d'éclatement puis d'unification patronale, en accordant une attention particulière à l'activité des créateurs des syndicats et à la manière dont elle s'articule avec les injonctions étatiques (liste des principaux sigles et acronymes en annexes).

\section{ENCADRÉ 1}

\section{Méthode et terrain}

Cette recherche a été menée dans le cadre d'un post-doctorat questionnant les spécificités des relations de travail dans les entreprises associatives et l'émergence de la fonction employeur.

Lors de notre enquête sur le CNEA, nous avons interrogé quinze personnes entre 2019 et 2020. Cinq d'entre elles furent à la tête de ce syndicat patronal, et/ou des organisations qui l'ont précédé dans les années 1980 à 2000, en tant que dirigeants salariés ou élus du conseil d'administration. Nous avons complété ce travail d'enquête par une série d'entretiens et d'échanges avec quatre membres de la direction actuelle de l'organisation, ainsi qu'avec quatre membres de syndicats patronaux partenaires (membres de l'UDES) et deux membres de syndicats de salariés.

En complément de ces entretiens, nous avons également étudié la littérature grise produite par les syndicats d'employeurs et de salariés de la branche de l'animation et quelques rares archives. Celles-ci étant particulièrement pauvres, notre travail repose essentiellement sur l'histoire orale transmise par les principaux protagonistes. Ce travail s'appuie aussi sur des entretiens menés entre 2011 et 2014, par Matthieu Hély, Maud Simonet et Romain Pudal, avec des dirigeants patronaux associatifs. Avec l'accord de ces chercheurs, nous avons mobilisé, dans cet article, trois de ces entretiens, réalisés avec des fondateurs du CNEA (que nous avons également rencontrés) qui interrogeaient l'émergence et le rôle des syndicats d'employeurs du secteur.

Pupilles de l'enseignement public (PEP), de la Fédération française de danse (FFD), de la Fédération des conservatoires d'espaces naturels, de la Fondation Nicolas-Hulot pour la nature et l'homme, de France nature environnement (FNE), de la Fédération Rhône-Alpes de protection de la nature (FRAPNA), du Groupe de réflexion, d'action et d'initiative pour la nature, l'environnement et la solidarité (GRAINES), de la Ligue pour la protection des oiseaux (LPO), du World Wildlife Fund (WWF), de la Fédération unie des auberges de jeunesse (FUAJ), de la Ligue de l'enseignement, de l'Union française des centres de vacances (UFCV), etc. 


\section{La création de la branche de l'animation : influence de la puissance publique et intérêt des entreprises associatives}

Nous avons repéré trois éléments qui ont servi de terreau à la création de l'espace patronal de l'animation. Les deux premiers sont des conséquences de l'action publique, à savoir la salarisation du secteur de l'animation et l'injonction à la création d'une branche professionnelle. Le dernier élément correspond à un besoin de régulation du secteur exprimé à la fois par les principales entreprises associatives et les syndicats de salariés.

\section{Une salarisation sans professionnalisation (1944-1980)?}

Historiquement, le secteur de l'animation socioculturelle est né d'un « mouvement social fondé sur le bénévolat et le militantisme et animé par des instituteurs, des prêtres, des dames patronnesses, des syndicalistes, des militants politiques et autres acteurs de l'intermédiation » (Augustin, GiLlet, 2000, p. 16). Il fait figure de prolongement professionnalisé de ces mouvements d' «éducation populaire ( Mignon, 2007; CORTESERO, 2012), qui ont une forte composante bénévole et viennent à la fois de la sphère religieuse et de la sphère laïque.

Ainsi, ce secteur s'est longtemps structuré en dehors de l'intervention de l'État. Cependant, à partir de 1936, la « jeunesse » devient une préoccupation gouvernementale (Augustin, Ion, 2017) et un domaine d'intervention publique (PALluaU, 2013). L'action étatique, qui correspond d'abord à une logique hygiéniste et médicale (LEBON, 2005), s'inscrit ensuite dans un projet global d'avenir de la société nationale. Comme l'explique la sociologue Patricia LONCLE (2003), pour le gouvernement du Front populaire (1936-1938), et son sous-secrétaire d'État aux Loisirs et aux Sports, Léo Lagrange, il s'agit de reconstruire un pays délabré et de fonder une société nouvelle.

Des politiques d' «encadrement» de la jeunesse sont mises en œuvre. Pour ce faire, l'État ne va pas intervenir directement, mais s'appuie sur le monde associatif : il apporte son soutien aux organisations de jeunesse, qu'elles soient ouvrières (DUCOMTE et al., 2013) ou confessionnelles. Dans les années 1950, le soutien public à ces structures loi 1901 est grandissant: la jeunesse est devenue une priorité politique avec l'accroissement du nombre de jeunes baby-boomers (Mignon, 1998), la hausse de la délinquance juvénile et la médiatisation des blousons noirs (BESSE, 2008). Dans les années 1960, notamment dans le cadre de la loi-programme de 1961 élaborée par Maurice Herzog, d'importants financements visent au développement des équipements sportifs et socio-éducatifs (comme les Maisons des jeunes et de la culture ${ }^{4}$ ). À cette politique volontariste de «multiplication» des infrastructures s'ajoute un

4. On comptait au début de la $V^{e}$ République 172 Maisons des jeunes et de la culture (MJC). Elles sont un peu plus de 900 en 1968 (BEsse, 2008). 
soutien financier apporté aux acteurs associatifs ainsi que la création de « diplômes sous l'autorité de l'État et du monde associatif » (LEBON, 2009, p. 5), autant d'éléments qui vont participer à la salarisation (DuSSUET, FLAHAULT, 2010), c'est-à-dire au développement de l'emploi, de ce secteur d'activité.

Le nombre d'animateurs varie selon les sources, qui, en raison de la diversité des statuts et des nombreux contrats occasionnels, n'aboutissent pas aux mêmes résultats. Selon Jean-Pierre Augustin et Jean-Claude Gillet (2000), en 1980, l'Institut national de la statistique et des études économiques (Insee) estime entre 25000 et 33700 le nombre d'animateurs salariés permanents. « Une évaluation de l'Observatoire des professions de l'animation avance le chiffre de plus de 50000 animateurs en 1985 et estime à plus de 300000 le nombre de salariés dans le secteur de l'animation » (Augustin, Gillet, 2000, p. 107). Au début des années 1980, le secteur de l'animation est donc déjà « un monde du travail » (HÉLy, 2009, p. 12 et suivantes) marqué par l'importante présence de salariés ${ }^{5}$.

Si, à partir des années 1960, le secteur de l'animation a connu une importante salarisation, « le renforcement de la professionnalisation reste limité » (AUGUSTIN, GILLET, 2000, p. 63). En effet, comme le notent Jean-Pierre AUGUSTIN et Jean-Claude GILLET (2000, p. 63), l'espace social de l'animation est encore flou, il y a un « éclatement des formations » et celles-ci manquent de reconnaissance « puisqu'il reste possible d'être animateur sans posséder de diplômes » et sans détenir de contrat de travail. Qui plus est, au début des années 1980, la profession est peu organisée. Le dialogue social entre salariés et employeurs reste rare dans les entreprises associatives de l'animation, peu d'entre elles ont un accord d'entreprise, et le secteur ne dispose pas de convention collective nationale (MignON, 1998).

\section{Les lois « Auroux »: le dialogue social, résultat d'une obligation étatique}

En 1981, la gauche arrive au pouvoir avec un projet de «généralisation de la couverture conventionnelle » (TALLARD, 1990). Comme l'expose le ministre du Travail, Jean Auroux, dans le rapport qu'il rend en septembre 1981, tous les travailleurs doivent bénéficier d'un statut collectif négocié, une volonté politique qui débouche, l'année suivante, sur les lois « Auroux ». Celles-ci prévoient une obligation annuelle de négocier sur les salaires et le temps de travail, tant au niveau de l'entreprise que de la branche (JOBERT, 2000). Elles visent une extension de la négociation collective et la couverture de l'ensemble des secteurs par des branches professionnelles disposant de conventions collectives ${ }^{6}$.

\footnotetext{
5. Il continuera par la suite son développement. Depuis 2017, il compterait presque 200000 animateurs et directeurs (LEBON, 2020), et plusieurs centaines de milliers de jeunes animant les centres de loisirs et les centres de vacances (LEBON, 2009).

6. La proportion de salariés d'entreprises de plus de dix salariés couverts par une convention collective de branche est ainsi passée de $80 \%$ en 1981 à plus de $86 \%$ en 1986 (BENVENISTE, 1987).
} 
Répondant à la décision «par le haut» (COHEN, 1986, p. 267) de structurer en branches professionnelles les secteurs d'activité qui ne le sont pas encore, le ministère du Travail se rapproche des principaux acteurs de l'éducation populaire et organise les premiers pas du dialogue social de la branche de l'animation socioculturelle. Comme l'explique Yves Mouton (cité par Antoine JACOB, 2008 p. 14), fonctionnaire du ministère du Travail, qui présida la commission mixte paritaire de la future branche de l'animation dans les années 1980, à cette époque, «l'État était soucieux de faire en sorte qu'il y ait des normes du travail, qui s'appliquent, y compris dans ce champ-là » . Ce que confirme Henri Borentin, membre de la direction des Francas et qui participa, côté employeur, au dialogue social de la branche de l'animation à ses débuts :

«Le ministère était déterminé à ce que tous les secteurs importants soient couverts par des conventions collectives ${ }^{7}$. Et ils ont convoqué les partenaires sociaux à des rencontres préparatoires pour créer une branche professionnelle. »

(Entretien du 23 janvier 2020)

\section{Volontés associatives et syndicales pour réguler le secteur de l'animation}

L'injonction à la création d'une branche professionnelle rencontre l'intérêt de plusieurs entreprises associatives de l'animation, qui y voient une opportunité pour réguler le secteur et le cadre d'emploi. Car si les entreprises associatives de l'éducation populaire profitent de la dynamique de salarisation, elles n'en ont pas le monopole. Ces organisations se trouvent même en concurrence avec « une nébuleuse de structures fonctionnant sans autres règles que celles du droit du travail classique », pour reprendre les propos de Yves Mouton (cités par JACOB, 2008, p. 16). De plus, au début des années 1980, les principales têtes de réseaux associatives (Ligue de l'enseignement, Centre d'entrainement aux méthodes d'éducation active [CEMÉA], Francas, Fédération Léo-Lagrange, etc.) disposent d'accords d'entreprise régissant les relations avec leurs salariés (JАCOB, 2008). Ces accords, diversement appliqués par les structures locales, sont calqués sur les règles et les grilles de rémunération de la fonction publique $^{8}$. Or, comme l'explique Robert Baron ${ }^{9}$, dans un entretien du 3 février 2020,

7. La mise en place d'une convention collective est possible depuis la loi du 25 mars 1919. Qui plus est, la loi du 24 juin 1936 précise que celle-ci peut être impérativement applicable à tous les salariés d'une profession si elle a été conclue par « les organisations syndicales les plus représentatives » et qu'elle a fait l'objet d'un « arrêté d'extension » signé par le ministre du Travail. «Par un acte de l'autorité publique, la convention, négociée par des parties privées - représentants de salariés et des employeurs - devient la loi de la profession. Par ce mécanisme de l'extension, toujours en vigueur, les pouvoirs publics donnent par délégation un pouvoir normatif qui a l'apparence d'un contrat » (BEVORT, JOBERT, 2011, p. 101). Enfin, la loi du 13 juillet 1971 complète les dispositions précédentes et détermine « le droit des salariés à la négociation sociale collective de l'ensemble de leurs conditions de travail et d'emploi et de leurs garanties sociales ». 8. De nombreux bénévoles et travailleurs des associations de l'animation sont issus de l'Éducation nationale. C'est le cas notamment des fonctionnaires « mis à disposition » par ce ministère pour travailler dans les associations de l'éducation populaire. Leur expérience du travail et des conditions de travail au sein du service public sert de référence pour la structuration professionnelle du secteur associatif de l'animation.

9. Ancien animateur formé à la Ligue de l'enseignement, il fut secrétaire général de la fédération des œuvres laïques de Meurthe-et-Moselle pendant vingt ans. Secrétaire général de l'Union nationale des organismes de développement 
qui a négocié l'accord d'entreprise de la Ligue de l'enseignement de 1974 en tant que salarié avant de participer à la structuration de la branche côté employeur à la fin des années 1980, à ce moment-là, « les grandes associations considéraient [...] qu'elles ne pouvaient plus vivre sur des accords d'entreprise, qui sont des accords d'entreprise de la fonction publique adaptée ». Ces textes garantissent en effet de nombreux droits aux salariés (indemnité de résidence, avancement à l'ancienneté, évolution des salaires liée à l'inflation, etc.) que les associations ont alors des difficultés à mettre en œuvre en raison des financements publics en baisse ou moins dynamiques. Robert Baron précise que l'idée des fédérations associatives n'est pas de simplement dénoncer les accords d'entreprise, mais de mettre en place des règles pour l'ensemble du secteur de l'animation:

«Ces organisations, qui sont rattachées à des mouvements, demandent qu'il y ait un corps commun de normes. Et la convention nationale de l'animation est un peu née de ça, c'est-à-dire de l'idée d'essayer de structurer un secteur et de donner des codifications, une colonne vertébrale assez forte. [Pour que] quand il y a concurrence, les règles du jeu soient les mêmes. »

(Entretien du 3 février 2020)

Le souhait qu'ont les associations de définir des normes d'emploi et de travail rejoint celui des syndicats de salariés. Dans les années 1960 à 1980, la reconnaissance des métiers et des compétences des animateurs ainsi que le développement de leurs droits sociaux (rémunération, horaires, congés, etc.) font partie des revendications des syndicats de salariés, tout comme la mise en place d'une convention collective (Mignon, 2006). Cependant, ceux-ci « se battent pour la reconnaissance de leur profession [...] en ordre relativement dispersé » (Mignon, 1998, p. 280). Qui plus est, l'animation est, selon le syndicat Union nationale des syndicats CGT des personnels des associations et organismes sociaux, sportifs et culturels (USPAOC-CGT), marquée par la « faiblesse » des syndicats de salariés (PeYRE, 2005, p. 14), qui résulte en partie d'une particularité du secteur - le recours massif aux temps partiels et aux contrats saisonniers, véritables obstacles à la syndicalisation (PIGNONI, 2016) - à laquelle s'ajoute la diversité des statuts dans les associations de l'éducation populaire au sein desquelles les travailleurs ne sont pas tous salariés. Dans les années 1980, outre les bénévoles et les objecteurs de conscience, le secteur bénéficie du travail de plusieurs milliers de fonctionnaires « mis à disposition» ou détachés de leur administration (souvent des enseignants de l'Éducation nationale, cf. supra note 8). Une telle diversité de statuts alimente la fragmentation syndicale, les enseignants mis à disposition adhérant généralement aux syndicats de l'Éducation nationale, comme la Fédération de l'Éducation nationale (FEN). De plus, les frontières de l'animation, profession naissante, peuvent être floues, oscillant entre bénévolat et emploi. Comme le rappelle Francis

social, sportif et culturel (UNODESC), il a fait partie des pionniers du dialogue social dans le secteur de l'animation côté employeurs. Il deviendra délégué général du CNEA en 2005 et sera également président d'Uniformation, principal organisme paritaire collecteur agréé (OPCA) de l'économie sociale et solidaire. 
LEBON (2006), si la dichotomie entre animation bénévole et animation professionnelle date des années 1960 et 1970, «l'animation volontaire » (c'est-à-dire réalisée par les bénévoles) est parfois l'antichambre de l'animation professionnelle. Et le passage de la posture de militant ou de bénévole à celle de salarié semble être davantage un obstacle à la syndicalisation qu'un moteur pour celle-ci: « Les deux postures, celle du militantisme syndical et celle du militantisme associatif, que chaque animateur porte peu ou prou en lui, se heurtent dans un conflit idéologique interne qu'il lui faut dépasser, car c'est une position trop ambivalente pour être supportable » (MignON, 1998, p. 161).

À la fois salariés et militants/bénévoles, les animateurs ont des difficultés à se reconnaître comme des travailleurs, salariés d'une profession, et n'hésitent pas, pour la « cause » (le projet que porte et incarne l'association), à faire des heures supplémentaires non rémunérées, bénévoles (COTTIN-MARX, 2020; 2021b). Un tel flou est un véritable obstacle à leur syndicalisation.

\section{Émergence, éclatement et unification de l'espace patronal de l'animation}

Nous avons vu dans la partie précédente que le secteur de l'animation a connu une importante salarisation dans l'après-guerre, que l'État a enjoint aux entreprises associatives du secteur de créer une branche professionnelle et que cette injonction a rencontré l'intérêt de celles-ci. Ces éléments vont amener les entreprises associatives à s'organiser au sein de syndicats d'employeurs destinés à structurer la future branche professionnelle et à négocier au sein de cette dernière.

Nous nous intéressons ici à l'émergence de cet espace patronal. Nous verrons tout d'abord qu'au début des années 1980, celui-ci s'est scindé en deux, autour de l'opposition entre associations « confessionnelles », adhérentes du Syndicat des associations de développement culturel et social (SADCS), et « laïques » qui ont créé le Syndicat national des organisations gestionnaires d'activités éducatives et culturelles (SNOGAEC). Ensuite, nous étudierons l'éclatement de ces deux structures en 1988. Refusant que les animateurs de colonies de vacances entrent dans le droit commun du Code du travail, certaines associations « confessionnelles » lancent une nouvelle organisation patronale, le Syndicat national des employeurs de la formation et de l'animation (SNEFA), tandis que les associations « laïques » fondent quant à elles l'Union nationale des organismes de développement social, sportif et culturel (UNODESC). Enfin, nous tenterons de comprendre ce qui a poussé ces organisations à se rassembler dans un syndicat patronal unique au début du XXI ${ }^{e}$ siècle (frise chronologique en annexes). 


\section{Tensions entre associations laïques et confessionnelles}

Pour participer aux négociations de la branche professionnelle naissante (encadré 2, pour une présentation des enjeux de périmètre) au début des années 1980, le secteur de l'animation n'est pas dépourvu de syndicat employeur. Il en compte un: le SADCS, créé en 1971 dans le sillage de la loi «Delors » du 16 juillet 1971, portant sur « l'organisation de la formation professionnelle continue dans le cadre de l'éducation permanente ». Ce syndicat regroupe plusieurs entreprises associatives de l'éducation populaire (DESSERTINE et al., 2004) : la Fédération Léo-Lagrange, l'Union française des centres de vacances et de loisirs (UFCV), les guides et scouts de France, le Comité protestant des centres de vacances (CPCV) et une multitude de petites structures (JАСOB, 2008). Cette organisation était alors, pour Jacques Guénée ${ }^{10}$, qui en fut l'un des dirigeants en tant que secrétaire général de la Fondation Léo-Lagrange, «plus une amicale d'employeurs qu'un véritable syndicat. [...] On n'avait pas de représentant dans les instances paritaires, ni de relations avec les ministères de tutelle » (entretien du 12 février 2020). Avec l'ouverture du dialogue social, cette « amicale » va cependant sortir de sa léthargie et prendre part à la création de la branche professionnelle de l'animation, au sein de laquelle le syndicat jouera un rôle actif, précisément en matière de dialogue social.

Pour se distinguer des organisations qu'elles cataloguent comme « confessionnelles » ${ }^{11}$, les associations « laïques » de l'éducation populaire (Pupilles de l'enseignement public, CEMÉA, Francas, etc.) fondent en 1982 le SNOGAEC, dont la présidence est confiée à Maurice Bricourt de la Ligue de l'enseignement. Comme l'explique Henri Borentin, qui a été secrétaire général adjoint du syndicat avant d'en prendre la tête en 1988:

«Au début des années 1980, le ministère du Travail réunissait les partenaires sociaux. C'est à ce moment-là qu'on s'est rendu compte que le monde laïc serait exclu de ces discussions puisque le seul syndicat existant était le SADCS. C'était le seul qui allait être convoqué à ces décisions. [...] On a donc décidé de créer un syndicat, que l'on n'osait pas appeler patronal à l'époque, d'employeurs entre acteurs de la jeunesse en plein air, dont le nom sera le SNOGAEC.»

(Entretien du 23 janvier 2020)

10. Jacques Guénée fut membre du syndicat de salariés Force ouvrière au sein de la Fédération Léo-Lagrange. Secrétaire général de la Fédération Léo-Lagrange de 1979 à 1985, il est à ce titre membre du SADCS.

11. Certaines organisations comme la Fondation Léo-Lagrange sont membres du SADCS sans pour autant pouvoir être qualifiées de « confessionnelles » (cf. supra). 


\section{ENCADRÉ 2}

\section{Périmètre incertain, périmètre contraint de la branche de l'animation}

Comparativement à certains secteurs associatifs, comme le sanitaire et social ${ }^{1}$, le dialogue social s'est mis en place assez tardivement dans l'animation. Il a réellement démarré sous l'impulsion des lois « Auroux » qui ont obligé les employeurs et les salariés à mettre en place des négociations collectives de branche.

Cette obligation laissait cependant le champ libre aux organisations syndicales (de salariés et d'employeurs) pour décider du périmètre de sectorisation de leur champ professionnel. C'est ainsi que le Syndicat national des associations employeuses de personnel au service des centres sociaux et socioculturels, le SNAECSO (fondé en 1971 et renommé depuis Employeurs du lien social et familial [ELISFA]) signe le 4 juin 1983, avec la CFTC, la CFDT, FO et la CGT, la convention collective nationale des acteurs du lien social et familial. Étendue par arrêté du 22 janvier 1987, elle couvre les centres sociaux et socioculturels, les associations d'accueil de jeunes enfants et les associations du développement social local. Comme l'explique Robert Baron, qui participe au dialogue social dans le cadre de la convention collective nationale de l'animation côté employeurs de la fin des années 1980 aux années 2000:

«Les centres sociaux ont créé un champ conventionnel avant celui de l'animation. On peut imaginer que si l'animation avait tiré avant, il n'y aurait pas deux champs [...], mais parce qu'historiquement les centres sociaux, cinq ans avant, ont créé leur champ, aujourd'hui [en 2019] il y en a deux. »

(Entretien du 3 février 2020)

Alors que les métiers et les activités exercés par les entreprises associatives de la branche du lien social et familial et ceux de la future branche de l'animation sont parfois les mêmes, deux branches indépendantes ont pourtant été créées, au gré du jeu des acteurs. Ainsi, si l'État a impulsé le dialogue social dans la branche de l'animation, il n'en a pas défini les contours, laissant aux organisations syndicales le soin de préciser son périmètre (sans pour autant leur donner la possibilité d'empiéter sur une branche voisine déjà existante).

Pour créer la convention collective nationale de l'animation, les organisations syndicales et patronales doivent donc s'interroger - qu'est-ce que l' animation socioculturelle? et ne pas retenir les activités déjà couvertes, comme le tourisme social et familial doté d'une convention collective nationale depuis 1979, les foyers de jeunes travailleurs (1982) et les centres sociaux et socioculturels (1983).

1. Le secteur sanitaire et social a connu un développement salarial important plus précoce que l'animation et a mis en place des conventions collectives nationales dès les années 1950-1960. 


\section{Divisions patronales sur l'Annexe II du projet de convention collective nationale et création de la branche}

Dans la première moitié des années 1980, le dialogue social se met en place au sein d'une « commission mixte paritaire ${ }^{12}$ » (les débats sont animés par un représentant du ministère du Travail), les deux syndicats patronaux, sans organisation faîtière pour les encadrer, ayant face à eux les différents syndicats de salariés (CFDT, FO, CGT et, de manière plus épisodique, CFTC et CFE-CGC ${ }^{13}$ ). Deux entités de la CGT sont présentes, l'USPAOC-CGT ${ }^{14}$ et la Fédération de l'éducation, de la recherche et de la culture (FERC-CGT), les syndicats de salariés s'étant, eux aussi, construits autour d'activités et de métiers, et non autour de branches (qui n'existent pas encore). Robert Baron, qui assista aux négociations pour le SNOGAEC, raconte:

« Une discussion se met en place, qui prend quand même plusieurs années parce qu'il n'y a pas de repères, c'est-à-dire que là aussi le dialogue social se fait sans référence à l'époque. En plus, ceux qui mènent objectivement la négociation pour les patrons sont plutôt des gens qui ont des racines salariées. Ce sont des militants, parfois très engagés politiquement [...] ou alors ayant été engagés syndicalement. Donc c'est un patronat un peu particulier. [...] La discussion n'est pas facile parce qu'il n'y a pas de cadrage patronal clairement identifié et puis il n'y a pas non plus de grands mandats des organisations associatives: pour elles, c'est une découverte le dialogue social. »

(Entretien du 23 février 2011)

Les négociations avancent mais achoppent sur un point précis, le régime des personnels pédagogiques des centres de vacances et de loisirs. Les « animateurs de colos », souvent jeunes, «étaient mobilisables 24 heures sur 24 pendant trois-quatre semaines d'été, moyennant quelques francs par jour, en plus du gîte et du couvert. Ce régime particulier ne répondait en rien au Code du travail » (JACOB, 2008, p. 25). Le fonctionnaire du ministère du Travail, qui préside alors la commission mixte paritaire de la future branche de l'animation, propose un compromis, qui prend la forme de l' «Annexe II » du projet de convention collective nationale. Cette annexe prévoit notamment que ces animateurs occasionnels soient payés au forfait (deux heures pour une journée de travail).

Du côté des organisations de salariés, cette proposition rencontre le refus de la CGT en raison de son caractère dérogatoire au droit commun, mais aussi de certaines fédérations associatives, comme la Ligue de l'enseignement (MARTIN, 2016) et l'UFCV, pour qui le statut de ces animateurs, qui interviennent pendant les petites

\footnotetext{
12. Le sujet a été développé, en particulier dans l'entretien réalisé par Maud Simonet et Matthieu Hély avec Bernard Maurin de la Direction générale du travail, notamment en charge de la commission mixte paritaire de l'animation. 13. CFDT: Confédération française démocratique du travail; FO : Force ouvrière; CGT : Confédération générale du travail; CFTC: Confédération française des travailleurs chrétiens ; CFE-CGC: Confédération française de l'encadrement - Confédération générale des cadres.

14. L'Union des syndicats des personnels de l'animation et des organisations sociales sportives et culturelles est membre de la Fédération des syndicats CGT du spectacle.
} 
ou les grandes vacances scolaires, ne doit pas s'appuyer sur le droit du travail ${ }^{15}$. En effet, pour ces deux organisations, dont les colonies de vacances représentent une part importante de l'activité, être animateur est un « engagement » qui doit rester un acte purement volontaire. La crainte de ces grandes associations, dont l'action repose en partie sur le travail bénévole, est en réalité que le droit commun ne s'applique à l'ensemble des statuts d'animateurs, remettant en cause la viabilité financière des colonies de vacances.

Ce conflit entraîne une scission au sein même des deux syndicats d'employeurs. Du côté des mouvements d'éducation populaire « laïcs », la Ligue de l'enseignement, alors membre du SNOGAEC, fonde l'UNODESC. Du côté des mouvements « confessionnels », l'UFCV quitte quant à elle le SADCS pour créer le SNEFA. Cependant, malgré les contestations des deux nouveaux syndicats d'employeurs, la convention collective nationale de l'animation est signée le 28 juin 1988. Au départ de la Ligue de l'enseignement du SNOGAEC, Henri Borentin devient le secrétaire général de ce dernier. Il explique: « J'ai instruit le dossier d'extension. On l'a demandé car on considérait que le texte était assez bon pour être étendu, et on ne pouvait pas se permettre de créer une concurrence déloyale » (entretien du 23 janvier 2020). La demande est acceptée par le ministère du Travail et la convention collective nationale est « étendue » par l'arrêté du 10 janvier 1989: elle s'applique à toutes les entreprises associatives qui entrent dans le périmètre de la branche, c'est-à-dire même à celles qui ne l'ont pas signée comme la Ligue de l'enseignement et l'UFCV. L'UNODESC entreprend alors un recours contre cette « extension » décidée par le ministre du Travail mais n'obtient pas gain de cause.

Pour les deux nouveaux syndicats d'employeurs, un second enjeu émerge: se faire reconnaître comme syndicats représentatifs afin de pouvoir être présents et peser dans les négociations de branche (MAGGI-GERMAIN, 2012), mais aussi afin de participer à la gestion de la formation professionnelle, bénéficier des financements paritaires, etc. Alain Cordesse $^{16}$, qui présida l'UNODESC, raconte:

«Quand on est sorti du SNOGAEC, le nouveau syndicat s'est mis en quête de gagner sa représentativité, ils faisaient adhérer les associations [de la Ligue de l'enseignement] pour montrer qu'il y avait beaucoup d'adhérents. »

(Entretien du 29 janvier 2020)

L'UNODESC obtient la reconnaissance de sa représentativité cinq ans plus tard, ce que n'a pas cherché à faire le SNEFA, qui est resté, nous dit Robert Baron avec humour, « une amicale sympathique, qui n'a jamais pu peser sur les négociations » (entretien du 2 février 2020).

15. UNODESC (2006), Rapport moral et d'activité.

16. Alain Cordesse préside l'UNODESC à compter de 1995, puis le CNEA à partir de 2001. En 2007, il prend la tête de l'Union de syndicats et groupements d'employeurs représentatifs dans l'économie sociale (USGERES, qui change de nom en 2013 et devient l'Union des employeurs de l'économie sociale et solidaire [UDES]). 


\section{Nouvelles divisions autour des « 35 heures » et rassemblement stratégique}

Une seconde crise entre les trois syndicats d'employeurs représentatifs de la branche de l'animation (SADCS, SNOGAEC et UNODESC) est déclenchée par la loi «Aubry » d'orientation et d'incitation relative à la réduction du temps de travail du 13 juin 1998. Celle-ci prévoit le passage du temps de travail hebdomadaire de 39 à 35 heures et stipule que la mise en place de cette réforme nécessite un accord au niveau de la branche. Or, si celui-ci est bel et bien trouvé, il est signé par trois organisations de salariés (Fédération nationale des syndicats du spectacle de l'audiovisuel et de l'action culturelle CGT, CFDT, CFTC), mais par seulement une organisation patronale représentative: le SNOGAEC. Comme l'explique Robert Baron, qui est en 1999 trésorier de l'UNODESC:

« Tout le monde était d'accord pour les 35 heures, tous les employeurs, sauf qu'on n'était pas d'accord sur la façon d'y aller. Parce que dans le secteur animation, mais c'est valable plus largement, il n'y a pas de contrepartie, c'est-à-dire que quand vous baissez le temps de travail vous ne pouvez pas mécaniser grand-chose... On est sûr de l'intervention au niveau de la personne. On expliquait, au niveau de l'UNODESC, en se moquant un peu du SNOGAEC qui signait cet accord-là, que quand on projette un film, on ne pouvait pas le faire tourner plus vite. [...] On avait un vrai problème parce que passer à 35 heures, c'était donner une augmentation de plus de $11 \%$ aux salariés, sans contrepartie. On devait alors soit baisser la durée d'intervention ou on allait faire monter le coût des prestations. On a eu un débat extrêmement dur là-dessus. »

(Entretien du 23 février 2011)

L'accord, entre les organisations de salariés et le SNOGAEC, ne nécessite pas l'unanimité du côté des syndicats d'employeurs : il est donc validé par le ministère du Travail et étendu par arrêté le 10 janvier 1999.

À la suite de cette nouvelle crise au sein de l'espace patronal de l'animation, les représentants des syndicats d'employeurs constatent que leurs divisions jouent contre leurs intérêts. Alain Cordesse, de l'UNODESC, raconte: « Les guerres que nous menions étaient picrocholines, servaient à animer les réunions. Mais, avant même le clash des 35 heures, on sentait déjà une volonté de mieux collaborer » (entretien du 29 janvier 2020). Les syndicats patronaux, qui siègent par ailleurs ensemble au sein du collège employeur de l'OPCA (organisme paritaire collecteur agréé) Uniformation et de l'Union de syndicats et groupements d'employeurs représentatifs dans l'économie sociale (USGERES), décident donc, en l'an 2000, de créer une « union » entre les syndicats en fondant le Conseil national des employeurs associatifs (CNEA). Le premier objectif est de bâtir une organisation permettant aux syndicats d'employeurs de tenir une position commune et cohérente dans les négociations face aux syndicats de salariés. Le second est de construire un syndicat prestataire « de services » (GIRAUD, Healy, 2015) et de mettre en commun les ressources, notamment juridiques, dont disposent les syndicats d'employeurs. L'idée est non seulement de mutualiser les 
ressources mais aussi de produire les mêmes interprétations du droit du travail de la branche pour que leurs adhérents puissent s'en emparer.

Le CNEA joue effectivement son rôle de coordination car, à compter de la création de cette union, les trois syndicats patronaux représentatifs (auxquels s'ajoute le SNEFA) adoptent une position commune lors des sessions de dialogue social, alors que du côté des cinq syndicats irréfragables de salariés les désaccords persistent lors des négociations. Le succès de la structure de coordination va aboutir en 2005 : les syndicats patronaux lancent un chantier pour créer une structure unique. Il débouchera trois ans plus tard par la fusion des trois organisations en une seule, avec des adhésions et un service juridique unique.

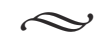

« Notre culture, c'est le regroupement », explique David Cluzeau, qui occupe le poste de délégué général du CNEA en 2020 (entretien du 10 mars 2020). Depuis 2005, le syndicat patronal n'a cessé de se renforcer et de s'agrandir, notamment par le biais de fusions avec d'autres syndicats patronaux tels que le Syndicat des associations de tourisme, de promotion sociale, de vacances et de loisirs (SATPS) en 2015 et le Syndicat national employeur des foyers, résidences sociales et services pour jeunes travailleurs (SNEFOS) en 2017. En 2018, le Syndicat national des organismes de formation (SYNOFDES) se rapproche du CNEA par, selon le communiqué de presse, « une convention d'association qui tout en respectant l'indépendance politique et juridique des deux organisations patronales mettra en synergie les atouts et expertises qui leur sont propres ». Ces rapprochements permettent au CNEA, devenu Hexopée en décembre 2020, d'asseoir sa représentativité au-delà de la branche de l'animation, dans les domaines du sport, du tourisme social et familial et des foyers et services pour jeunes travailleurs.

Le syndicat employeur de la branche de l'animation est ainsi devenu une force centripète dans le champ patronal associatif. De plus, ces réorganisations successives préfigurent la définition de futurs champs professionnels au moment même où l'État impulse une restructuration des branches professionnelles ${ }^{17}$.

Lors d'un entretien réalisé début 2020, Yann Poyet, syndicaliste de Force ouvrière, qui participe depuis les années 1980 au dialogue social de la branche de l'animation et qui est à ce titre un bon connaisseur des organisations professionnelles qu'il a face à lui, explique: «En 1980 comme en 2020, les syndicats d'employeurs se retrouvent dans la même situation. Ils n'avaient pas forcément envie de faire ce genre de choses. C'est

17. Depuis les années 2000, plusieurs rapports commandés par les gouvernements successifs appellent au regroupement des branches professionnelles. C'est le cas du rapport Virville (2004), du rapport Poisson (2009), des rapports Combrexelle (2013 et 2015), du rapport Quinqueton (2015) qui vont être suivis de plusieurs lois. La loi du 5 mars 2014, du 8 août 2016 et les ordonnances « Macron » de 2017 donnent à l'État des moyens juridiques pour obliger les branches à se regrouper et s'assurer de la mise en œuvre de ces fusions. L'objectif est de passer de 925 branches professionnelles existantes fin 2016 à moins d'une centaine à terme (rapport Ramain, version de travail, février 2020). 
poussés par l'État qu'ils ont pris les choses en main. » La démonstration réalisée dans cet article rejoint cette analyse. L'État a joué un rôle crucial dans la structuration de l'espace patronal de l'animation, qui semble être le résultat des réactions associatives à une succession de « crises » provoquées par la puissance publique: salarisation du secteur et injonction à l' ouverture du dialogue social, mise en place d'une branche professionnelle couvrant l'ensemble des salariés, nécessité de mettre en œuvre la réforme des 35 heures, fusion des branches professionnelles, etc. L'agenda social, imposé par l'État, a impulsé ses transformations. Ainsi, faire la genèse du CNEA montre, encore une fois, que pour faire la sociologie du monde associatif, et plus particulièrement celle du monde du travail associatif dont ces employeurs font partie, il est nécessaire de ramener «l'État dans l'analyse » (Evans et al., 1985; SimONET, 2010, p. 81). Son action a été déterminante pour que ces employeurs « engagés » que sont les dirigeants associatifs, dépassent leurs réticences, pour qu'ils acceptent de prendre en charge la fonction employeur qui leur incombe et pour qu'ils s'organisent au sein de syndicats patronaux en capacité de participer à la structuration institutionnelle d'une branche professionnelle.

Cependant, le processus qui a abouti à l'unification de l'espace patronal de l'animation dans un syndicat employeur unique, solidement établi, n'était pas écrit d'avance. L'étude de la fondation du CNEA permet en effet de mettre en exergue les logiques propres au champ patronal associatif et de souligner les concurrences (parfois historiquement héritées) entre organisations patronales et entreprises associatives du secteur, ainsi que les tensions idéologiques et les intérêts divergents qui les traversent. Elle montre un monde du travail associatif sous pression de l'État, mais avec une histoire et des intérêts qui lui sont spécifiques.

\section{BIBIIOGRAPHIE}

Augustin J.-P., Gillet J.-C. (2000), L'Animation professionnelle. Histoire, acteurs, enjeux, Paris, L'Harmattan.

Augustin J.-P., Ion J. (2017), Loisirs des jeunes. 120 ans d'activités éducatives et sportives, Paris, La Documentation française.

BENVENISTE C. (1987), «Les négociations salariales: de la convention collective à l'accord d'entreprise », Économie et Statistique, n ${ }^{\circ}$ 199-200, pp. 19-23.

BESSE L. (2008), Les MJC, 1959-1981. De l'été des blousons noirs à l'été des Minguettes, Rennes, Presses universitaires de Rennes.

BeVORT A., JOBERT A. (2011), Sociologie du travail. Les relations professionnelles, $2^{\mathrm{e}}$ éd., Paris, Armand Colin.

Bunel J., Saglio J. (1979), L'Action patronale. Du CNPF au petit patron, Paris, Presses universitaires de France. 
Caillaud P. (2012), « Devenir une organisation représentative au niveau national interprofessionnel: les enjeux juridiques de l'action des employeurs de l'économie sociale », Travail et Emploi, $\mathrm{n}^{\mathrm{o}}$ 131, pp. 47-64.

CArdoso A. (2019), «Quand les patronnes sont bénévoles: conflictualités au Planning Familial », La Nouvelle Revue du travail [en ligne], n ${ }^{\circ}$ 15. https://doi.org/10.4000/nrt.5780

Chauvière M. (2005), « Pour une genèse de la branche professionnelle sanitaire, sociale et médico-sociale, à but non lucratif », Communication à Unifaf Auvergne, 16 juin.

CoHEn E. (1986), « Le “moment lois Auroux” ou la désublimation de l'économie », Sociologie du travail, vol. 28, $\mathrm{n}^{\mathrm{O}} 3$, pp. 265-285.

CORTESERo R. (2012), «Éducation populaire et animation », Fiches Repères, nº 9, Institut national de la jeunesse et de l'éducation populaire.

Cottin-Marx S. (2020), «Les relations de travail dans les entreprises associatives. Salariés et employeurs bénévoles face à l'ambivalence de leurs rôles », La Revue de l'Ires, n 101-102, pp. 29-48.

COTTIN-MARX S. (2021a), « Être employeur bénévole dans une crèche associative et parentale », Le Sociographe, $\mathrm{n}^{\mathrm{0}}$ 73, pp. 53-65.

Cottin-Marx S. (2021b), C'est pour la bonne cause! Les désillusions du travail associatif, Ivry-sur-Seine, Éditions de l'Atelier.

Cottin-Marx S., Hély M. (2021), « Naissance d'un patronat "responsable et engagé". L'économie sociale et solidaire en quête de représentativité patronale », Cadres, $\mathrm{n}^{\circ} 488$, pp. 13-22.

Dessertine D., Durand R., Eloy J., Gardet M., Marec Y., Tétard F. (dir.) (2004), Les Centres sociaux 1880-1980. Une résolution locale de la question sociale?, Villeneuve-d'Ascq, Presses universitaires du Septentrion.

Ducomte J.-M., Martin J.-P., Roman J. (2013), Anthologie de l'éducation populaire, Toulouse, Privat.

DussuEt A., Flahault É. (2010), « Entre professionnalisation et salarisation, quelle reconnaissance du travail dans le monde associatif? », Formation emploi, n ${ }^{\circ} 111$, pp. 35-50.

Evans P., Rueschemeyer D., Skocpol T. (1985), Bringing the State Back In, Cambridge, Cambridge University Press.

Fleuriel S. (2013), « Ce que dialoguer veut dire. L'émergence du “dialogue social” dans le sport français (1990-2006) », Genèses, n ${ }^{\circ}$ 92, pp. 127-146.

FLEURIEL S. (2016), «Le sport professionnel saisi par sa convention collective: genèse d'une définition singulière (France, 1995-2014) », Le Mouvement social, n 254, pp. 133-144.

FLEURIEL S. (2021), La Convention collective nationale du sport. Un droit sur mesure, Fontaine, Presses universitaires de Grenoble.

FRAIZY J.-J. (2016), «La structuration politique de l'ESS à l'épreuve du territoire. Analyse d'une organisation départementale de représentation de l'ESS », RECMA, n 339, pp. 71-84. 
Giraud B., Healy A. (2015), « Le syndicalisme patronal comme syndicalisme de services: l'offre de services dans le travail de recrutement local des patrons », Sociétés contemporaines, $\mathrm{n}^{\mathrm{o}}$ 98, pp. 19-48.

Hély M. (2009), Les Métamorphoses du monde associatif, Paris, Presses universitaires de France.

Hély M., Pudal R., Simonet. M. (2014), « “Autre économie”, autre démocratie sociale ? Les organisations patronales entre syndicats patronaux de l'économie sociale et solidaire et champ politique », in Fraboulet D., Humair C., Vernus P. (dir.), Coopérer, négocier, s'affronter. Les organisations patronales et leurs relations avec les autres organisations collectives, Rennes, Presses universitaires de Rennes, pp. 289-300.

JАСОв A. (2008), Petite Histoire de la branche de l'animation. Les partenaires sociaux racontent, Paris, Éditions Branche professionnelle de l'animation.

JOBERT A. (2000), Les Espaces de la négociation collective, branches et territoires, Toulouse, Octarès Éditions.

Jobert A. (2012), « Patronat », in Bevort A., Jobert A., Lallement M., Mias A. (dir.), Dictionnaire du travail, Paris, Presses universitaires de France, pp. 526-532.

Lebon F. (2005), Une politique de l'enfance. Du patronage au centre de loisirs, Paris, L'Harmattan.

LEBOn F. (2006), « De “nouvelles professions” entre précarité et flexibilité : animateurs socioculturels et formateurs d'adultes (1982-2002)», Regards sociologiques, $\mathrm{n}^{\circ} 32$, pp. 83-95.

Lebon F. (2009), Les Animateurs socioculturels, Paris, La Découverte.

LEBON F. (2020), Entre travail éducatif et citoyenneté : l'animation et l'éducation populaire, Nîmes, Champ social.

Le Roy A., Puissant E. (coord.), Devetter F.-X., Vatan S. (2019), Économie politique des associations. Transformations des organisations de l'économie sociale et solidaire, Louvainla-Neuve, De Boeck supérieur.

LONCLE P. (2003), L'Action publique malgré les jeunes. Les politiques de jeunesse en France de 1870 à 2000, Paris, L'Harmattan.

Maggi-Germain N. (2012), «Fonctions et usages de la représentativité patronale », Travail et Emploi, $\mathrm{n}^{\mathrm{o}} 131$, pp. 23-45.

Maggi-Germain N., Le Crom J.-P., Caillaud P., Carré S., Héas F., Blatge M., Chauchard J.-P., Saglio J. (2011), La Construction de la représentativité patronale [Rapport de recherche], Paris, Direction de l'animation de la recherche, des études et des statistiques (Dares).

Marchal E. (1989), La Professionnalisation des associations, Thèse en sociologie, Université Paris 5.

MARCHAL E. (1992), «L'entreprise associative entre calcul économique et désintéressement », Revue française de sociologie, vol. 33, n 3. pp. 365-390.

Martin J.-P. (2016), La Ligue de l'enseignement. Une histoire politique (1866-2016), Rennes, Presses universitaires de Rennes. 
Michel H., Willemez L. (2009), « Les employeurs de l'économie sociale : des patrons comme les autres ? Une enquête aux prud'hommes », Savoir/Agir, n ${ }^{\circ} 10$, pp. 33-41.

Mignon J.-M. (1998), La Lente Naissance d'une profession: les animateurs, de 1944 à 1988, Thèse en histoire contemporaine, Université Michel-de-Montaigne Bordeaux III.

Mignon J.-M. (2006), « Quand les salariés d'associations de tourisme social, de loisirs et d'animation socioculturelle se syndiquèrent à la CFDT, février 1964-décembre 1968 », in Tartakowsky D., Tétard F., Syndicats et associations. Concurrence ou complémentarité?, Rennes, Presses universitaires de Rennes, pp. 181-189.

Mignon J.-M. (2007), Une histoire de l'éducation populaire, Paris, La Découverte.

OFFERLÉ M. (2009), Sociologie des organisations patronales, Paris, La Découverte.

OFFERLÉ M. (2015), « Militer en patronat. Engagements patronaux et sociologie du militantisme », Sociétés contemporaines, n ${ }^{\circ}$ 98, pp. 79-106.

OfFERLÉ M. (2018). «Chapitre 2. Les représentativités patronales », in Andolfatto D., La Démocratie sociale en tension, Villeneuve-d'Ascq, Presses universitaires du Septentrion, pp. 47-66.

Palluau N. (2013), La Fabrique des pédagogues. Encadrer les colonies de vacances, 19191939, Rennes, Presses universitaires de Rennes.

Peyre M. (dir.) (2005), Le Livre noir de l'animation socioculturelle, Paris, L'Harmattan.

Pignoni M. T. (2016), «La syndicalisation en France. Des salariés deux fois plus syndiqués dans la fonction publique », Dares analyses, $\mathrm{n}^{\circ} 025$.

Poujol G. (1981), L'Éducation populaire : histoires et pouvoirs, Paris, Éditions ouvrières.

Simonet M. (2010), Le Travail bénévole. Engagement citoyen ou travail gratuit?, Paris, La Dispute.

Simonet M. (2015), «Carrière de permanent. De militant à employeur ou les enjeux de l' “omnipositionnalité” », in Camus J., Lebon F., Regards sociologiques sur l'animation, Paris, La Documentation française, pp. 85-99.

TALlard M. (1990), « La généralisation de la couverture conventionnelle », Travail et Emploi, $\mathrm{n}^{\mathrm{o}} 46, \mathrm{pp} .15-22$.

TChernonog V., Prouteau L. (2019), Le Paysage associatiffrançais. Mesures et évolutions, $3^{\mathrm{e}}$ éd., Paris, Dalloz.

UrasadetTAn J., Schmidt C. (2020), «Les pratiques RH au sein des petites associations de l'économie sociale et solidaire: défaut, déni ou défi d'employeurabilité ? », @GRH, n 36 , pp. 97-117.

Woll C. (2006), «La réforme du Medef: chronique des difficultés de l'action collective patronale », Revue française de science politique, vol. 56, n ${ }^{\circ}$ 2, pp. 255-279. 


\section{ANNEXES}

\section{Liste des principaux sigles et acronymes}

\section{Syndicats d'employeurs}

CNEA : Conseil national des employeurs associatifs (qui se nommera ensuite Conseil national des employeurs d'avenir avant de prendre le nom d'Hexopée fin 2020).

SADCS : Syndicat des associations de développement culturel et social (syndicat « confessionnel », favorable à l'Annexe II).

SNEFA: Syndicat national des employeurs de la formation et de l'animation (syndicat « confessionnel », défavorable à l'Annexe II).

SNOGAEC: Syndicat national des organisations gestionnaires d'activités éducatives et culturelles (syndicat « laïc », favorable à l'Annexe II).

UDES : Union des employeurs de l'économie sociale et solidaire (anciennement Union de syndicats et groupements d'employeurs représentatifs dans l'économie sociale, USGERES).

UNODESC: Union nationale des organismes de développement social, sportif et culturel (syndicat « laïc », défavorable à l'Annexe II).

\section{Associations}

CNAJEP: Comité pour les relations nationales et internationales des associations de jeunesse et d'éducation populaire.

CPCV : Comité protestant des centres de vacances.

UFCV : Union française des centres de vacances et de loisirs. 
FRISE CHRONOLOGIQUE - Évolutions patronales et interventions publiques

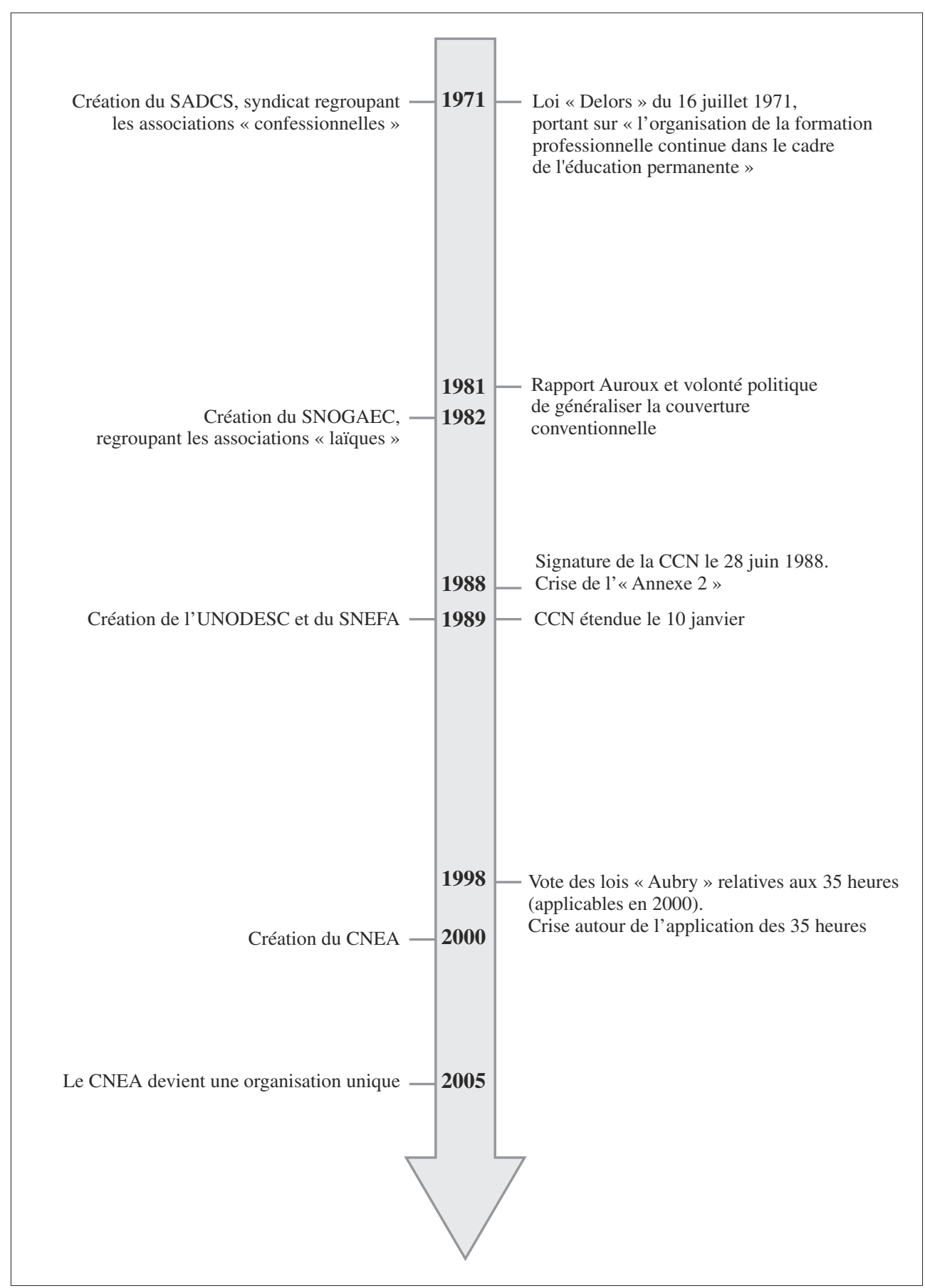


